\title{
Research on maintenance tools of wire clamps for live working on Ultra High Voltage transmission lines
}

\author{
Zhigang Wang ${ }^{123}$, Wenjian Mei ${ }^{123}$, Qi Yang ${ }^{123}$, Mingming Qiao ${ }^{123}$ Jianping Chen $^{123}$ and Peng $\operatorname{Li}^{123 *}$ \\ ${ }^{1}$ Live Line Operation Center, Power Transmission Maintenance Branch of State Grid Hunan Electric Power Co., Ltd., ChangSha, Hunan, \\ 410000, China \\ ${ }^{2}$ Intelligent live working technology equipment (robot) Hunan Provincial Key Laboratory, ChangSha, Hunan, 410000, China \\ ${ }^{3}$ Live inspection and intelligent operation technology State Grid Corporation Laboratory
}

\begin{abstract}
Aiming at the problem that the suspension clamp is difficult to be quickly replaced due to the heavy load of the suspension clamp when replacing the transmission line wire suspension clamp in the UHV live operation, this paper develops an auxiliary device for realizing the rapid replacement of the conductor suspension clamp. Through the design of fixtures suitable for the installation of UHV wires and connecting plates, the load transfer of the suspension clamp can be quickly realized, and the suspension clamp can be separated and replaced under the condition of no force. The device is flexible and light, and effectively improves the live working personnel work efficiency.
\end{abstract}

\section{Introduction}

The Qishao $\pm 800 \mathrm{kV}$ UHV DC transmission line is an important power transmission project for the country's "West-East Power Transmission". Its line spans the 5 provinces of Gansu, Shaanxi, Chongqing, Hubei and Hunan, with a total length of 2,383 kilometers. The corridors of the line are complicated. Some lines have long been tested by severe weather such as sandstorms, high temperatures, blizzards, and severe cold. With the commissioning and long-term operation of Qishao UHV transmission lines, transmission line failures continue to increase, and the demand for maintenance devices for live work is also increasing.

Suspension clamps are commonly used fittings on transmission lines, and their main function is to fix the wires on the insulator string of the linear tower. During the long-term operation of the line, the suspension clamp is exposed to the natural environment for a long time, and is constantly subjected to various severe weather tests such as strong winds and sandstorms, and the probability of failure continues to increase [1]. At present, the load of the entire set of wires is usually transferred with the aid of cross arm clamps, wire rods, insulated pull rods and wire hooks. The tools are heavy, the transfer load is large, the operation is difficult for the operators, and it takes a lot of time and physical strength, which brings great safety Risk $[2,3]$. [2,3]. In response to this problem, a "UHV transmission line wire suspension clamp fixture" was developed to transfer only the required sub-conductor load to efficiently complete the live replacement of the UHV transmission line suspension clamp work.

\section{2 requirements of the design}

According to the functional requirements, this article designs each part of the suspension clamp fixture, which is divided into the design of the board card, the design of the tightener part, and the overall design. Finally, the mechanical calculation is used to check whether the tool meets the requirements. The specific design idea is shown in Figure 1.

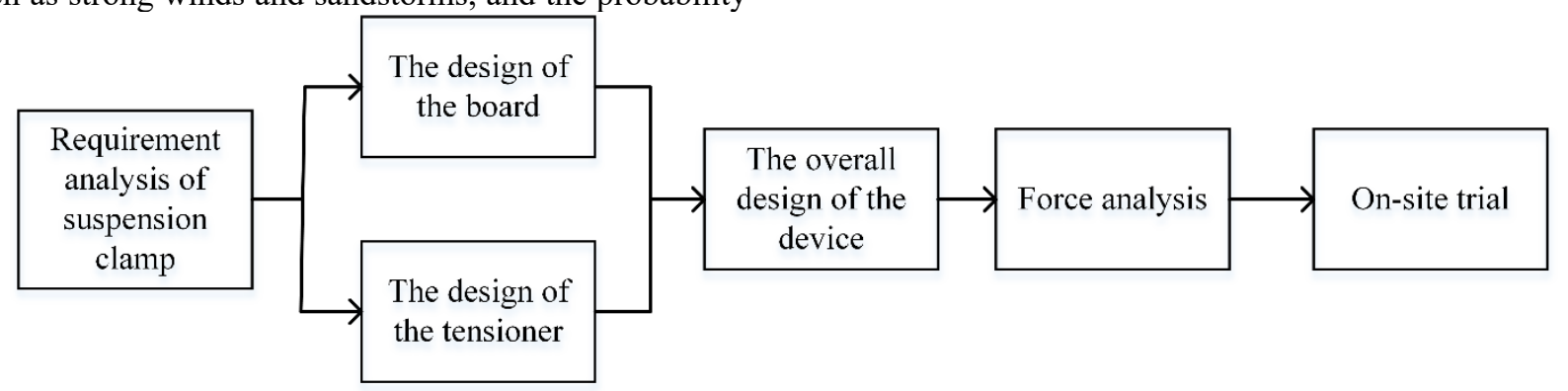

Figure 1 Design ideas of suspension clamp 
The specific instructions are as follows

(1) Analyze the structure of the connecting plate, the suspension clamp and the conductor, and design a fixture that can replace the suspension clamp only by transferring the required sub-conductor load;

(2) Analyze the stress on the middle and lower lines of the transmission line, and design corresponding components according to the requirements;

(3) Compare the maximum line load of Qishao Line with the mechanical load of the tool, and redesign if it does not meet the requirements;

(4) The complete set of tools includes the board clamp, the wire tensioner bracket, the suspension screw, the support screw and the wire fixing frame, and its design, development, improvement and manufacturing must meet the "State Grid Corporation Electric Safety Work Regulations Line Part" And the relevant provisions of the "Basic Requirements for Design and Testing of Transmission Line Construction Equipment".

\section{Device configuration and processing}

\subsection{Composition and principle}

UHV transmission line wire suspension clamp fixture is composed of joint board clamp, wire tensioner bracket, screw rod and wire fixing frame. Use the tie-board card as a fixed holder, and use tools such as the wire tensioner bracket, screw rod, and wire fixing frame to lift the wire. The device uses the connecting plate as a support point to lift a single wire, and each end of the connecting plate has a set of devices. Therefore, the force of a single screw is only half of the load of the replaced sub-wire, and the tool takes up less space and the safety factor is greatly improved.

\subsection{Union board design}

The main function of the connecting board is to connect the tensioner bracket and the connecting board, so that it can move synchronously. The symmetrical midplane of the traditional big knife chuck surface has a through slot with a thickness that matches the connecting plate of the circuit fittings. The connecting plate of the wire is closely attached to the through slot, and the knife edge forms a mechanical fulcrum through the connecting plate bolt, but the UHV circuit is connected The board space structure is complicated, and the practicability is not high. Therefore, the design of the flat card as shown in Figure 2. The connecting board uses bolts 1 to fix the flat board on the connecting board, and the reserved screw holes 2 are used to fix the wire tensioner bracket, with stable connection, flexible disassembly and assembly, and strong reliability.

\subsection{Tighter bracket design}

Based on the design of the tie-board card and the tensioner, it is necessary to design a tensioner bracket that can fix the tensioner on the tie-board card to ensure that the tensioner can firmly press during the tightening process. On the connecting board, the designed cable tightener bracket is shown in the figure. The analysis shows that the wire tensioner bracket is fixed on the coupling plate by bolts, and the screw rod is fixed by the tenon joint.

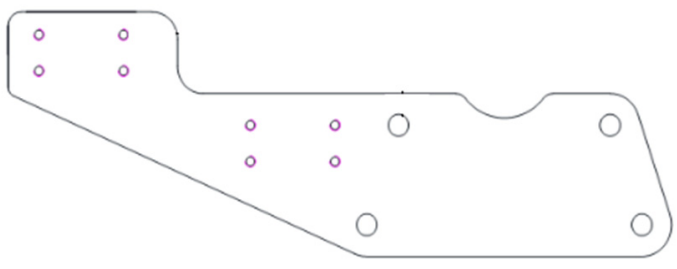

Figure 2 Schematic diagram of connecting board

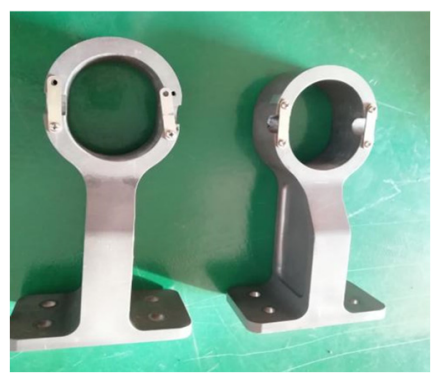

Figure 3 Schematic diagram of the tensioner bracket

\subsection{Wire tensioner}

Due to the limitation of the connecting board structure, the connecting board card and the wire tensioner bracket can only be erected in the middle of the connecting board. The stress situation of the upper, middle and lower wires is analyzed, and two different wire lifting methods and two wire fixing frames as shown in the figure are designed.

As shown in Figure 4(a), the support screw: used for load transfer on the upper wire, and closed support claws are used to prevent the wire from slipping out, and the safety is high; as shown in Figure 4(b), the suspension screw: used for middle and Under the wire load transfer, design the wire hook as shown in the figure 4. 


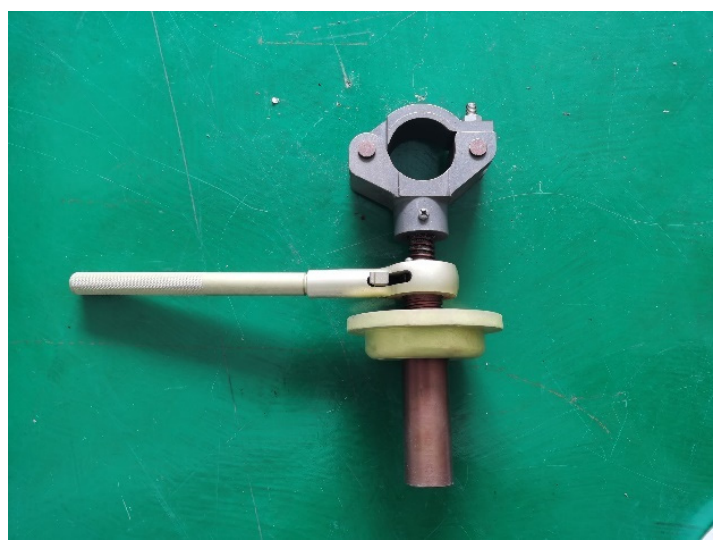

(a) Schematic diagram of support screw

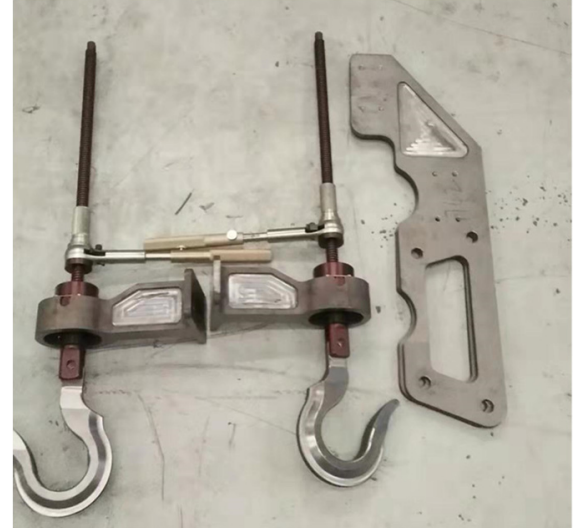

(b) Schematic diagram of suspension screw

Figure 4 Schematic diagram of the tightener

\section{Assembly test and application}

\subsection{Overall assembly}

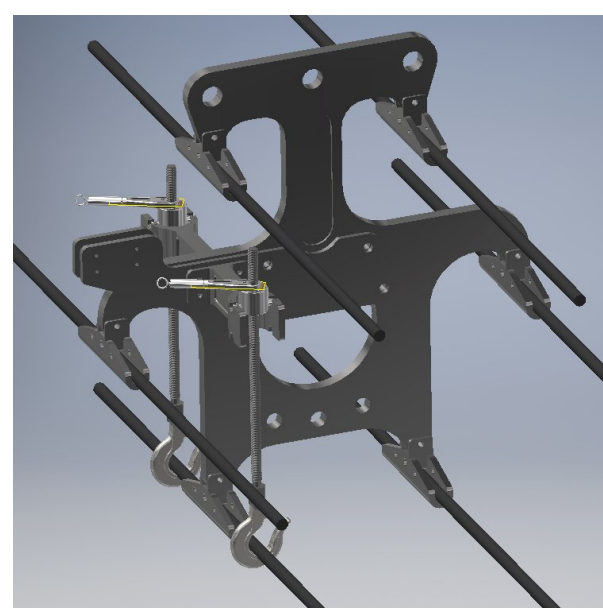

(a) Wire lifting type
The picture shows the assembly diagram of the new type of suspension clamp, which is divided into two types: (a) wire lifting type and (b) wire top type. As shown in the figure, the two styles are mainly composed of a connecting plate, a wire tensioner bracket, and a wire tensioner. Each part is connected by bolts and tenon joints.

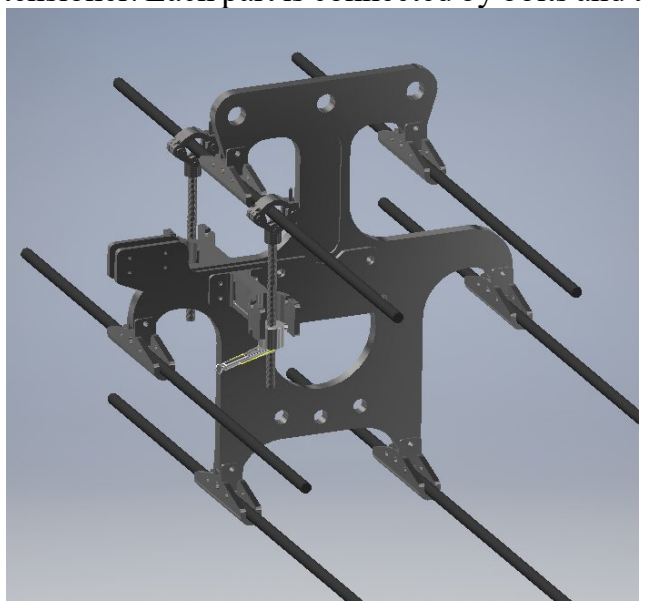

(b) Wire top type

Figure 5 Schematic diagram of overall assembly

\subsection{Mechanical calculation}

selected for calculation. The basic parameters of the selected line are:

According to the situation of the entire Qishao line, the line with the largest wire type and the largest line span is

Table 1 Wire parameters

\begin{tabular}{ll}
\hline \multicolumn{1}{c}{ parameter } & \\
\hline Wire model value \\
cross-sectional area $(\mathrm{mm} 2)$ & $\mathrm{JL} 1 / \mathrm{G} 2 \mathrm{~A}-1250 / 100$ \\
Outer diameter $(\mathrm{mm})$ & 1350.03 \\
Calculated weight $(\mathrm{kg} / \mathrm{km})$ & 47.85 \\
Calculate breaking force $(\mathrm{N})$ & 4252.3 \\
Span $(\mathrm{m})$ & 329.85 \\
Vertical span $(\mathrm{m})$ & 879 \\
\hline
\end{tabular}

It is carried out in good weather, without ice, and wind speed is not greater than 5 levels. Therefore, in the relevant calculations, only the weight of the wires and fittings and the load caused by wind pressure are considered. 


\subsection{Calculation and Analysis of Force on Linear Tower}

It is carried out in good weather, without ice, and wind speed is not greater than 5 levels. Therefore, in the relevant calculations, only the weight of the wires and fittings and the load caused by wind pressure are considered.

(1) Self-weight load of transmission wire

$$
g_{1}=\frac{G_{0}}{S}=30.86 \times 10^{-3}\left(\mathrm{~N} / \mathrm{m} \bullet \mathrm{mm}^{2}\right)
$$

$g_{0}$-wire weight per kilometer $(\mathrm{kg} / \mathrm{km}), S$ The entire cross-sectional area of the wire.

(2) Wind pressure $P$

The wind pressure on the wire is caused by air movement energy. The horizontal wind pressure in the vertical line direction is:

$$
P=\alpha K F \frac{V^{2}}{16} \sin ^{2} \theta(9.8 \mathrm{~N})=\frac{1 \times 1.1 \times 100 \times 47.85}{1.6 \times 1350.03}=24.36 \times 10^{-3}\left(\mathrm{~N} / \mathrm{m} \bullet \mathrm{mm}^{2}\right)
$$

$\alpha$-coefficient of uneven wind speed 1.0, wind speed $10 \mathrm{~m} / \mathrm{s}, F$-The projection surface of the wind surface on a plane perpendicular to the wind direction, $\theta$ The horizontal angle between the wind direction and the wind projection surface, When calculating, consider that the wind direction is perpendicular to the direction of the

$$
Q=g_{1} S l_{\mathrm{z}}+W=30.86 \times 10^{-3} \times 1350.03 \times 772+100 \times 9.8=33143.01 N
$$

power line $\sin ^{2} \theta=1, K$ - Aerodynamic coefficient 1.1.

(3) Comprehensive ratio

$$
g_{6}=\sqrt{g_{1}^{2}+g_{4}^{2}}=39.31 \times 10^{-3}\left(\mathrm{~N} / \mathrm{m} \bullet \mathrm{mm}^{2}\right)
$$

(4) Vertical load on the insulator string
Where $\mathrm{K}$ Including spacer rods, pull plates, wire clamps and the weight of the equipotential operator, it is approximately $200 \mathrm{~kg}$ (the weight of composite insulators is not considered, $\pm 800 \mathrm{kV}$ UHV lines are six-split conductors)

(5) Horizontal load on the insulator string

$$
P=g_{4} S l_{\mathrm{p}}=20422.66 \mathrm{~N}
$$

(6) The total load on the insulator string is

$$
G=\sqrt{Q^{2}+P^{2}}=38929.99 N \approx 38.9 k N
$$

(7) Total load on a single sub-conductor is

The load strength of a single hanging wire clamp fixture is $40 \mathrm{KN}$, and the load strength of the whole set of fixtures is $80 \mathrm{KN}$, which is much larger than the total load of a single wire, so it meets the operating requirements.

\subsection{On-site trial}

In order to verify the actual effect of the device, the live operation center of the State Grid Hunan Power Transmission Maintenance Company carried out the live replacement of the UHV transmission line simulation operation at the training site, and the following work flow was formulated:

(1) The ground electrician connects the wire fixing device with the screw rod in advance, and then successively transmits each part of the device to the electrician on the tower through the insulating rope;

(2) Two equipotential electricians first fix the connecting plate clamp on the wire connecting plate, then install the tensioner bracket and the tensioner one after another, then tighten the tensioner, and replace the wire clamp after the load transfer is completed.

\section{Conclusion}

The clamp fixture designed in this paper is used in the maintenance work of the live replacement of the UHV transmission line, and the suspension clamp operation time is reduced from 3 hours to 1.4 hours. It greatly reduces the labor intensity of the operators and improves the efficiency of equipment maintenance.

\section{Acknowledgments}

This article was funded by the special scientific research project of the Ministry of Science and Technology of the State Grid Hunan Electric Power Co., Ltd. "The method and equipment for live operation of Qishao Ultra High Voltage, 5216A318000R"

\section{references}

1. Yang Shiqiang, Weng Yongchun, Li Xiaolai, et al. Analysis and Research on Hidden Dangers of Uneven Ice Coating Damage to UHV DC Fittings[J]. Hubei Electric Power, 2018, 042(003): 5-8, 25.

2. Zhang Zisheng, Zhou Cheng, Huang Jing. Development and application effect analysis of live working tools for $\pm 800 \mathrm{kV}$ UHV DC transmission lines[J]. Science and Technology Innovation and Application, 2019(35).

3. Wu Zhengshu, Fan Changhai, Tao Huiying, et al. Analysis on the method of live replacement of the wire clamp of the linear tower of $\pm 800 \mathrm{kV}$ DC transmission line[J]. Guangxi Electric Power, 2014. 